\title{
Sistemas de información en red para estudiantes universitarios: marco conceptual y evaluación
}

\author{
Por Josep Cobarsí Morales
}

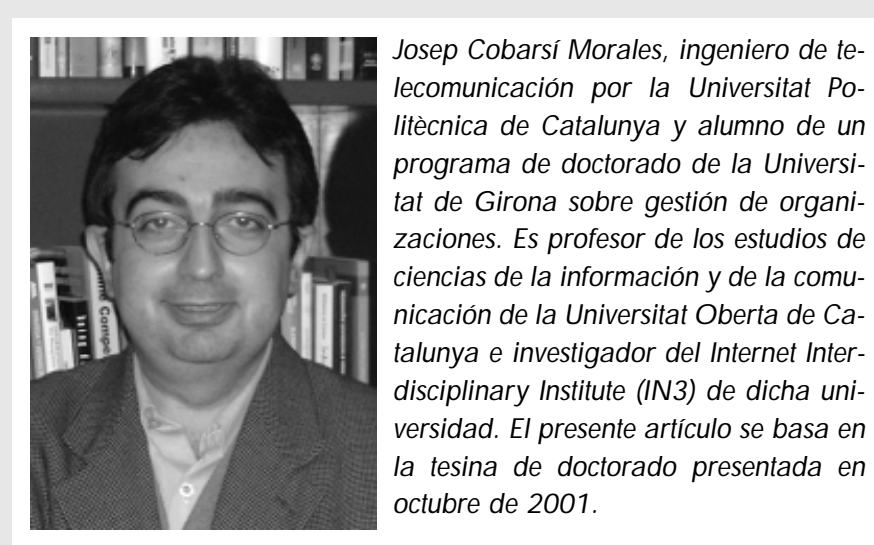

Resumen: Tras definir qué se entiende por sistema de información en red para estudiantes universitarios, se fijan los requisitos de dichos sistemas atendiendo a los cambios operados en las universidades y a las necesidades futuras de la enseñanza universitaria. A continuación se analizan las principales propuestas metodológicas y estudios realizados para la evaluación de dichos sistemas. Se finaliza ofreciendo algunos datos sobre la situación actual de dichos sistemas.

Palabras clave: Sistema de información, Sistema de información en red, Universidad, Estudiante, Evaluación, Entorno informacional, Intranet, Sistema de información de campus, Usuario.

\section{Title: Networked information systems for university students: conceptual framework and evaluation}

\begin{abstract}
After initially defining what is understood by a networked information system for university students, the requirements for such systems are set forth based on changes occurring at universities and on the future needs of higher education. The main methodological proposals and studies concerning system evaluations are then analysed. Finally, data are offered about the current state of these systems.
\end{abstract}

Keywords: Information System, Networked information system, University, Student, Evaluation, Information environment, Intranet, Campus-wide information system, User.

Cobarsí Morales, Josep. "Sistemas de información en red para estudiantes universitarios: marco conceptual y evaluación”. En: El profesional de la información, 2002, julio-agosto, v. 11, n. 4, pp. 256-263.

\section{Definición y contexto}

Basándonos en el concepto de sistema de información en línea de una universidad expresado por Charles R. McClure en su manual Assessing the academic networked environment: strategies and options (1996), proponemos la siguiente definición de sistema de información en red para estudiantes universitarios: "conjunto de contenidos y servicios, accesible por medios informáticos dentro de un entorno web, que una universidad pone a disposición de sus estudiantes para facilitar sus diversas actividades académicas, administrativas y sociales".

Respecto a esta definición, conviene resaltar las siguientes consideraciones:

-Queda abierta la tipología de entorno web. Así, el término "intranet" no reflejaría adecuadamente la situación real actual de los sistemas de información en red de muchas universidades. Hoy en día, buena parte de los recursos y servicios de información se encuentran en las webs exteriores de las instituciones universitarias, o accesibles desde ellas de forma restringida desde diversos puntos (aunque se tienda a su integración en una intranet de acceso restringido a los estudiantes y/o otros colectivos de usuarios de la propia institución).

-Identificamos entorno red con entorno web. En la literatura anglosajona se usa el término campus-wide information system (cwis) para designar, según su formulación original, al sistema de información en web o en gopher de una universidad ${ }^{1}$. Cuando aparece este concepto a principios de los años 90, el sistema de información en red universitario estaba constituido no solamente por un entorno gopher o web (un cwis), sino también por recursos en discos compartidos en red local. Dada la situación y tendencias actuales, en que los recursos y servicios de información tienden a pre- 
sentarse en un contexto web, podemos considerar este concepto como equivalente al de sistema de información en línea universitario. Y como tal se emplea en los artículos recientes de la correspondiente bibliografía especializada ${ }^{2}$.

- Consideramos la vida del estudiante universitario como una experiencia de formación integral, más allá de una mera yuxtaposición de asignaturas cursadas a lo largo de varios semestres.

- Si bien nuestra atención principal se dirige a las fuentes de información, debemos tener en cuenta la infraestructura informática. El acceso a dichos sistemas ha sido, históricamente, más difícil para los estudiantes que para el profesorado o personal de gestión. Y todavía es una cuestión pendiente de resolver ${ }^{3}$.

- La incorporación del usuario en el diseño y mejora del sistema de información es recomendable pero plantea diversas problemáticas ${ }^{4}$. Los estudiantes presentan a este respecto dificultades más acentuadas. En cambio, profesorado y personal de gestión son menos numerosos y tienen, en principio, una mayor facilidad de contacto y comunicación con los diseñadores del sistema.

\section{«Hoy en día, buena parte de los recursos y servicios de infor- mación se encuentran en las webs exteriores de las institu- ciones universitarias»}

Como veremos a continuación, las tendencias en el mundo universitario apuntan a cambios en el perfil de los estudiantes, especialmente a una mayor diversidad. Y ello debe tenerse en cuenta al proponer los requisitos de un sistema de información para estos usuarios.

\section{Resumen de tendencias en las universidades actuales}

Nos basamos principalmente en los informes "The virtual university" y "L'educació superior al segle XXI" ${ }^{\prime}$. En estos dos documentos, y demás bibliografía anotada en este apartado, parece existir un alto grado de acuerdo respecto a las grandes direcciones de cambio y a su impacto cualitativo a corto y medio plazo. Por el contrario, hay diversidad de opiniones entre moderados $^{7}$ y radicales $^{8}$ sobre la velocidad futura $\mathrm{y}$ consecuencias finales de los cambios.

Las principales tendencias que afectan al mercado laboral en relación a la formación son:

-Necesidades de aprendizaje a lo largo de la vida, debido a los procesos de reorganización de las empre$\operatorname{sas}^{9} \mathrm{o}$ al ritmo de aparición de nuevo conocimiento que obliga al reciclaje formativo individual de los emplea$\operatorname{dos}^{10}$.

- Demanda creciente de competencias en tecnologías de la información y de capacidades de trabajo en equipo.

-Aumento del teletrabajo, por lo menos durante una parte de la jornada laboral.

En cuanto a tendencias tecnológicas, podemos remarcar:

-Digitalización. En especial hay que tener en cuenta el incremento del ancho de banda y del parque informático disponible en domicilios particulares.

-Madurez. Se traduce en el uso de las tecnologías de la información para propósitos generales (procesado de textos, correo electrónico, etc.).

-Desintermediación. Muchas de las demandas de un estudiante universitario serán satisfechas por un sistema de información, sin intervención humana directa $^{11}$.

Acerca del impacto previsible de todos estos cambios a corto y medio plazo cabe señalar principalmente:

- Variación de la demografía estudiantil, con el incremento de estudiantes adultos, a quienes resulta imposible realizar su aprendizaje en un campus tradicional debido a diversos motivos (horarios, responsabilidades familiares, etc.). En el mundo anglosajón estos colectivos reciben el nombre genérico de nontraditional students ${ }^{12}$.

-Demanda creciente, a la cual contribuyen muy especialmente los nuevos colectivos de estudiantes mencionados.

-Presión para aumentar la productividad en docencia y servicios ante la previsible situación de una mayor demanda por un lado y, por otro, la disminución de recursos públicos.

-Presión para satisfacer las expectativas de los estudiantes, en particular respecto al acceso y uso de recursos de información en red ${ }^{13}$.

- Mayor poder de decisión de los estudiantes y menor peso de la geografía en la configuración de su currículo académico.

- Creación de nuevas formas de cooperación entre diferentes instituciones en relación con la enseñanza universitaria. Los participantes en este proceso no tienen por qué ser necesariamente estamentos universitarios, y puede tratarse también de empresas tecnológicas, editoriales, etc..$^{14}$.

Todo ello implica un incremento de la educación a distancia (a veces denominada virtual), en relación con 
el sistema tradicional de enseñanza (también llamado presencial ${ }^{15}$ ), combinándose a menudo ambas modalidades en una misma universidad, titulación o asignatura. En todo caso, y sean cuales sean las modalidades de docencia practicadas en cada institución, los servicios de información en línea tienen una importancia creciente. Un conjunto adecuado de contenidos y servicios en línea (no necesariamente relacionados con el mundo de la educación propiamente dicho), puede aportar sustanciales mejoras en la vida del estudiante universitario.

Otra importante implicación es la necesidad creciente de la evaluación universitaria, no solamente como instrumento de mejora interna, sino también dirigida al entorno exterior (poderes públicos, patrocinadores privados o potenciales estudiantes).

\section{Requisitos de un sistema de información en red universitario para estudiantes}

En coherencia con la definición anterior y las tendencias que acabamos de señalar, y a la vista de documentación y estudios recientes, podemos proponer una serie de contenidos y servicios. La variación de perfiles y necesidades permitiría añadir otros elementos y/o matizar su importancia. Pero al menos podemos establecer una mínima base de elementos comunes, susceptible de discusión y perfeccionamiento. Los elementos propuestos se basan principalmente en la guía de Educause (Educause consumer guide), lista de preguntas que un estudiante debe plantearse al buscar universidad. De la misma fuente hemos adoptado también la clasificación por apartados: ámbito académico, administrativo y social (quedan recogidos en la tabla 1). Casi todos los elementos se consideran igualmente en la última versión del ranking anual America's 100 most wired colleges and universities ${ }^{16}$, dirigido al mismo tipo de audiencia. Y aparecen también en la encuesta anual Campus computing survey ${ }^{17}$, destinada a gestores universitarios. Algunas universidades han publicado variantes de la guía Educause ${ }^{18}$. Si bien puede considerarse en términos generales un alto grado de coincidencia respecto a los contenidos y servicios susceptibles de ser incluidos, debemos constatar también:
- Los elementos mencionados son de muy distinto tipo (recursos de información, grupos de recursos similares, servicios) y no constituyen una lista exhaustiva de definiciones precisas.

-El punto de vista es el de un alumno norteamericano que, procedente de la enseñanza secundaria, busca universidad en su país para solicitar ser admitido, con la intención de residir en el campus durante los periodos académicos. La formulación podría necesitar diversas modificaciones y matices si cambia el punto de vista (por ejemplo: estudiante en un país europeo que busca universidad en su propio país, de un programa de intercambio, educación a distancia, etc.). En cualquier caso, no nos constan propuestas alternativas formuladas desde un contexto europeo.

- Para poder hablar con propiedad de sistema y caracterizarlo adecuadamente, debería tenerse en cuenta no solamente la presencia o no de ciertos contenidos y servicios individualmente considerados de potencial utilidad al usuario, sino también ciertas propiedades de la arquitectura de información del conjunto tales como: navegabilidad, recuperación de la información, coherencia gráfica y terminológica ${ }^{19}$. Éstas y otras características, relacionadas con la usabilidad del sistema, son soslayadas en los documentos mencionados.

Las referencias citadas tienen en cuenta, además de contenidos y servicios: la facilidad de acceso al sistema por parte de los estudiantes mediante recursos informáticos a su disposición, el soporte y formación al usuario, y cómo se afrontan los problemas de financia- 
ción que todo ello conlleva. Sin embargo, mientras para el caso de los contenidos y servicios puede establecerse una cierta base de elementos comunes, la diversidad de usuarios e instituciones suscita controversia sobre los demás aspectos ${ }^{20}$. Por ejemplo una cuestión muy importante es cómo garantizar un acceso adecuado al sistema por parte de los alumnos: aunque algunas instituciones recomienden o incluso exijan a sus estudiantes adquirir sus propios ordenadores portátiles, no hay acuerdo sobre si es la mejor alternativa.

\section{Modelos de evaluación de sistemas de información en red para estudiantes universitarios: propuestas metodológicas y estudios realizados}

Una vez introducido el concepto y requisitos básicos, vamos a hacer algunas consideraciones como marco de referencia respecto a la evaluación de estos sistemas, sobre aspectos tales como: colectivos interesados, caracterización y ámbito del estudio.

\section{«Una cuestión muy importante es cómo garantizar un acceso adecuado al sistema por parte de los alumnos: algunas institu- ciones anglosajonas exigen a sus estudiantes adquirir sus propios ordenadores portátiles»}

Los colectivos interesados pueden ser:

- Responsables de gestión estratégica para establecer planificación a medio y largo plazo, o para conocer en qué medida el sistema de información sirve a los objetivos y misión de su institución.

-Encargados de la gestión operativa para monitorizar y realizar, si es el caso, los ajustes necesarios en los servicios a su cargo.

-Estudiantes como elemento para establecer preferencia sobre en qué universidad o universidades desarrollar su currículo académico, o para conocer la situación actual en su institución.

- Responsables de políticas educativas a nivel de país para observar la situación y tendencias en el conjunto de instituciones universitarias.

En cuanto a los aspectos del sistema que pueden ser evaluados para su caracterización, puede considerarse en primer lugar su grado de completitud: qué contenidos o servicios existen y qué tipo de prestaciones ofrecen ${ }^{21}$. Otras posibles cuestiones, siguiendo la clasificación de McClure serían:

—Extensión: en qué medida se usa cierto servicio o recurso por parte de sus usuarios potenciales.
-Eficiencia: coste de los elementos necesarios para proporcionar servicios o recursos concretos.

-Eficacia: grado de cumplimiento de los objetivos planteados respecto a un programa o servicio.

- Calidad de servicio: hasta qué punto éste es suministrado según los parámetros establecidos.

-Impacto: beneficios más allá de lo inmediato, a nivel individual u organizacional, del uso de cierto servicio o recurso.

—Utilidad: en qué medida los servicios y recursos son útiles o apropiados para el usuario.

-Usabilidad ${ }^{22}$ : facilidad de uso. Cabe señalar que este aspecto es especialmente importante para el caso de los estudiantes, ya que acostumbran a tener recursos informáticos y tiempo limitado para acceder al sistema.

El ámbito de la evaluación puede ser intrainstitucional (una sola universidad) o interinstitucional (un conjunto de instituciones). Las primeras, realizadas con o sin ayuda del exterior, son útiles básicamente a la propia organización (o como referencia para otras que pretendan realizar un estudio similar). Las segundas pueden servir a estudiantes potenciales o a los responsables de políticas educativas (pero también es interesante para cada universidad conocer cuál es la situación general y su posición en el conjunto).

\section{Propuestas metodológicas.}

Para el caso de una evaluación intrainstitucional puede tomarse como referencia el manual de McClure "Assessing the academic networked environment: strategies and options“ que ya ha sido citado. Suministra definiciones muy precisas de más de 60 parámetros cuantificables sobre contenidos y servicios, infraestructura, soporte y gestión. Discute el uso de técnicas de recogida y tratamiento de datos cualitativos aplicados a la evaluación de estos sistemas. Señala asimismo las dificultades prácticas que pueden plantearse, como la dispersión entre distintas unidades y departamentos de los datos a recoger. Una referencia más reciente, y complementaria de la anterior, es "Institution-wide information management and its assessment" ${ }^{\prime 23}$, que analiza con detalle la gestión, planteando una lista de 25 preguntas para evaluarla. Finalmente, otro documento interesante (aunque es una referencia más antigua) es "Evaluation guidelines for institutional information resources", que establece un conjunto de concisas orientaciones generales para quien plantee por primera vez evaluaciones en una institución, propone además una lista de 45 preguntas. La formulación y clasificación de preguntas y definiciones es menos clara que en las otras dos propuestas ${ }^{24}$. 
Para el caso de una evaluación interinstitucional, podemos destacar dos problemáticas comunes en este tipo de estudios: la formulación de definiciones válidas para el conjunto de instituciones estudiadas y la elección de procedimientos de recogida de información fiable y contrastada. Como veremos en el siguiente apartado, algunos investigadores optan por pedir la colaboración activa de las instituciones estudiadas, mientras que otros recogen datos por procedimientos alternativos. A falta de bibliografía metodológica específica para este tipo de estudios, podemos señalar los apartados de la tesis mencionada en el siguiente apartado "Online student services in American higher education: contemporary issues and future implications".

Tanto si se trata de evaluaciones de ámbito intrainstitucional como interinstitucional, la bibliografía metodológica clásica disponible sobre técnicas de recogida y tratamiento de datos no contempla suficientemente su administración por vía telemática (por ejemplo, para el caso de las encuestas, su gestión mediante formularios $\mathrm{web}^{25}$ ). Esta carencia puede suplirse en parte gracias a un reciente manual de McClure y Bertot sobre evaluación de sistemas de información en línea no restringido al ámbito universitario sino de carácter general ${ }^{26}$.

En el caso de nuestro país, podemos citar el "Catálogo de indicadores del sistema universitario público", dentro del Plan Nacional de Evaluación ${ }^{27}$. De los 43 establecidos, solamente 2 pueden considerarse relacionados con el sistema de información en línea universitario, en concreto respecto a infraestructura técnica: número de ordenadores de uso público tanto en bibliotecas como en aulas informáticas.

Veamos a continuación algunos ejemplos de estudios realizados especialmente relevantes, ya tengan como ámbito un conjunto de instituciones o una sola. La mayoría de ellos se han realizado en universidades anglosajonas.

\section{Estudios realizados en el ámbito de un con- junto de instituciones.}

El estudio anual Campus computing survey ${ }^{28}$ (dentro del programa Campus computing project, iniciado en 1990) analiza el grado de implantación y uso de las tecnologías y recursos de información en campus, mediante encuesta a más de 600 instituciones universitarias norteamericanas. Trata de gestión, contenidos y servicios, formación y soporte, aunque presta mayor atención a infraestructura tecnológica, llevándose a cabo mediante encuesta. El cuestionario es muy exhaustivo y se remite a los responsables de los servicios informáticos y de gestión académica de cada institución. No solamente se preguntan datos sobre la situación ac- tual sino también sobre tendencias previstas para el futuro inmediato. El tratamiento de todos los datos es estadístico, tomando en consideración el tipo de institución. La presentación de los resultados completos de la encuesta está pensada para gestores universitarios, pero los datos de mayor interés para estudiantes pueden consultarse a través de la Educause consumer guide. Las estadísticas presentan la proporción de instituciones en relación con el número total, pero no tienen en cuenta las dimensiones de la institución.

El mencionado America's 100 most wired colleges and universities ${ }^{29}$ establece un ranking anual de los sistemas de información en red para estudiantes de las universidades norteamericanas. Los datos de la encuesta, mediante cuestionario a responsables de las universidades estudiadas, se recogen electrónicamente. Las sucesivas versiones anuales se han ido refinando incorporando sugerencias de estudiantes, padres y gestores universitarios. La rápida evolución de los sistemas también se ha tenido en cuenta para ir modificando los cuestionarios y pesos del ranking. La documentación disponible no justifica o discute la fórmula de elaboración de los rankings de cada año. Se limita a suministrar la puntuación asignada a cada institución, desglosada en diversos grandes apartados (en la encuesta 2001: recursos para estudiantes, portal, e-learning, soporte técnico, acceso inalámbrico). Es la única vía de obtención de datos y no consta que se efectúe comprobación de su exactitud.

\section{«En España se viene realizando anualmente desde 1994 la en- cuesta Rebiun sobre diversos aspectos de los servicios e in- fraestructura bibliotecaria de nuestras universidades»}

En la tesis "Online student services in American higher education: contemporary issues and future implications" $(1998)^{30}$, se analiza el grado de desarrollo de la oferta de servicios en red a los estudiantes (concretamente, en lo que respecta a: tutorización, inserción profesional, actividades sociales y culturales, finanzas, salud y prevención). Se centra, por lo tanto, en una parcela limitada del sistema de información para estudiantes. El tratamiento de datos es estadístico, según ciertas características de las universidades (tamaño, carácter público o privado) y no se realiza ninguna clasificación o ranking individual de las instituciones. Cabe señalar que la recogida de datos del trabajo de campo tuvo lugar exclusivamente mediante visita a los sitios web exteriores de las universidades (es decir, sin necesidad de una colaboración activa por parte de ellas y siquiera sin su conocimiento). Aunque esto puede 
contribuir a la fiabilidad de los datos recogidos, también es susceptible de acarrear desventajas en la medida que la tendencia a la creación de intranets limita el alcance de una exploración realizada desde el exterior. El trabajo no ha tenido continuidad posteriormente.

En nuestro país, la encuesta Rebiun (Red de Bibliotecas Universitarias Españolas) se viene realizando anualmente desde 1994 sobre diversos aspectos de los servicios e infraestructura bibliotecaria de nuestras universidades ${ }^{31}$. Uno de los datos recogidos es el número de terminales informáticas a disposición del público desde las bibliotecas. Dado el objetivo del estudio, no se contemplan otros datos necesarios para completar la visión sobre la infraestructura de acceso al sistema de información universitario, como puede ser por ejemplo el número de ordenadores en aulas informáticas.

\section{Estudios realizados en el ámbito de una sola institución.}

Podemos destacar los propiciados a finales de los años 90 por la Coalition for Networked Information en 6 instituciones norteamericanas y una británica). Cada una de ellas realizó un estudio limitado a ciertos elementos y aspectos de su sistema de información, aplicando las directrices del manual de McClure. En la mayoría de los casos se considera, además de los estudiantes, a otros usuarios:

—Evaluación del impacto de las actividades de formación ofrecidas por bibliotecas y aulas informáticas (Brown University).

—Evaluación de los servicios de "help desk" y soporte informático (Darmouth University).

-Uso y satisfacción de un servicio de distribución electrónica bajo demanda de material a los estudiantes realizado por el servicio de biblioteca (Gettysburg College).

—Evaluación del sistema de acceso a revistas electrónicas: uso, costes, perfiles de usuario y disponibilidad (King's College).

-Expectativas, experiencias $\mathrm{y}$ actitudes de profesorado de primer año y estudiantes de primer año respecto al entorno en red (Mary Washington College).

-Evaluación del programa piloto Uwired para la integración en la enseñanza de las tecnologías de la información y los recursos de información electrónica (University of Washington).

Cabe destacar que a través de algunos de estos estudios se constata la dificultad de calcular costes de infraestructura (procedente de diversas partidas presupuestarias de difícil identificación, a lo largo de diferentes ejercicios) o de acciones de soporte al usuario (realizadas por distintas unidades organizativas). Probablemente dichas dificultades son extrapolables también en el caso de universidades de otros países.

Para más información y referencias sobre metodología y otros detalles de dichos estudios véase el artículo de Lippincott "Assessing the academic networked environment" ${ }^{32}$. Podemos encontrar reports de otros estudios y exposiciones de casos en la página web de Educause (Educause information resources library $\left.^{33}\right)$.

\section{Sistemas de información en red para estudiantes universitarios: situación actual}

Finalizaremos con una primera aproximación a la situación y tendencia en los campus actuales en base a los datos disponibles recogidos por fuentes que se han citado a lo largo de este artículo.

En la tabla 2 constan datos recogidos en Campus computing survey 2000 sobre la presencia o no de ciertos contenidos y servicios del sistema de información para estudiantes en el ámbito de las universidades norteamericanas. El grado de presencia va desde la existencia consolidada de catálogos en línea, a la aparición todavía incipiente de servicios de comercio electrónico. No nos consta la existencia de datos similares en el ámbito de las universidades españolas o europeas, pe-

\begin{tabular}{|c|c|c|}
\hline & $\begin{array}{c}\text { Públicas } \\
\%\end{array}$ & $\begin{array}{c}\text { Privadas } \\
\%\end{array}$ \\
\hline \multicolumn{3}{|l|}{ Ámbito académico: } \\
\hline $\begin{array}{l}\text { - Información electrónica de soporte al curso (programa, } \\
\text { bibliografía). }\end{array}$ & 67,7 & 66,7 \\
\hline - Recursos de biblioteca: catálogo. & 98,5 & 95,2 \\
\hline $\begin{array}{l}\text { - Recursos de biblioteca: revistas electrónicas y bases de } \\
\text { datos. }\end{array}$ & 90,8 & 85,7 \\
\hline \multicolumn{3}{|l|}{ Ámbito administrativo: } \\
\hline - Acceso a sus datos personales: expediente académico. & 53,8 & 61,9 \\
\hline - Comercio electrónico: pago de facturas, pedidos en línea. & 40,0 & 33,3 \\
\hline - Matrícula y modificación de matrícula en línea. & 69,2 & 71,4 \\
\hline - Solicitud de acceso en línea para nuevos estudiantes. & 92,3 & 81,0 \\
\hline \multicolumn{3}{|l|}{ Ámbito social: } \\
\hline $\begin{array}{l}\text { - Información de contacto de profesores y personal de } \\
\text { gestión. }\end{array}$ & 96,9 & 95,2 \\
\hline
\end{tabular}

Tabla 2. Presencia de contenidos y servicios de un sistema de información en red para estudiantes en universidades norteamericanas. Porcentaje de instituciones públicas o privadas donde existen los contenidos y servicios. Fuente: Campus computing survey 2000. 


\begin{tabular}{|c|c|c|}
\hline $\begin{array}{l}\text { Ratio estudiantes/ordenadores de } \\
\text { uso público en bibliotecas }\end{array}$ & Número de universidades & $\%$ del total de estudiantes \\
\hline$<200$ & 15 & 21,0 \\
\hline $200-400$ & 18 & 35,4 \\
\hline $400-600$ & 16 & 29,9 \\
\hline$>600$ & 9 & 13,7 \\
\hline
\end{tabular}

ro podemos suponer que la situación aquí será en general de menor presencia de contenidos y servicios.

Sí disponemos de un dato sobre la infraestructura de acceso al sistema de información para el caso de las universidades españolas: la ratio de ordenadores de uso público en relación con el número de usuarios (tabla 3), en donde pueden observarse considerables desniveles entre instituciones. En todo caso sería necesario disponer de más datos para completar la información sobre infraestructura de acceso (por ejemplo, sobre ordenadores en aulas informáticas o propiedad de los estudiantes). Para el caso norteamericano tenemos algunos datos sobre la proporción de estudiantes que tienen su propio ordenador y sobre la proporción de instituciones que lo exigen a sus estudiantes, pocas pero que representan un porcentaje ya apreciable (tabla 4). Esta exigencia no nos consta en ninguna universidad de nuestro país, salvo en el caso de la Universitat Oberta de Catalunya donde los estudiantes deben proporcionarse uno, así como conexión a internet.

Una primera exploración de las webs exteriores de nuestras universidades en la actualidad, así como la lectura de textos sobre la situación de las instituciones norteamericanas a finales de la década de los 90, apunta a que los recursos de información en red acostumbran a surgir en un principio de manera bastante anárquica y dispersa. A menudo se detecta la presencia de ciertos elementos relacionados con la docencia o con los servicios universitarios, sin llegar a constituir un sistema que merezca tal nombre, aunque todas las instituciones tienden más o menos rápidamente a la creación de entornos integrados en forma de intranet. Ello es ya una realidad plena, por ejemplo, en la Universitat d'Alacant ${ }^{34}$, la Universidad de Almería ${ }^{35}$ o la Universitat Autònoma de Barcelona ${ }^{36}$.

\section{Conclusiones}

En un sistema de información para estudiantes deben tenerse en cuenta los recursos de información necesarios para sus diversas actividades académicas, administrativas y sociales, sin olvidar la arquitectura de información del conjunto y la infraestructura informática de acceso.

Se constata la necesidad creciente de evaluación en las instituciones universitarias tanto para su mejora interna como para información al exterior (poderes públicos, patrocinadores privados, potenciales estudiantes, etc.).

Algunas de las principales dificultades que se plantean para la evaluación de los sistemas de información académicos son: el hecho de que diversas unidades organizativas de una misma universidad tengan responsabilidades sobre ellos, su rápida evolución y la de encontrar definiciones válidas y no ambiguas para un conjunto de universidades. La infraestructura de acceso por parte de los estudiantes es una cuestión por resolver, y que afecta muy marcadamente a este tipo de usuarios.

Las diversas caracterizaciones sugeridas en todos los trabajos mencionados parecen basarse principalmente en el conocimiento acumulado por expertos, así como en la reflexión de éstos pensando en el usuario. Pero, en general, éste tiene pocas ocasiones de participar de forma activa y consciente en la elaboración de las caracterizaciones. Por tanto podría decirse, tal vez exagerando un poco, que "todo para el usuario pero sin el usuario".

Hay una gran disponibilidad por parte de las universidades norteamericanas a responder a preguntas sobre su sistema de información para estudiantes, tanto si se trata de encuestas procedentes del exterior de

\begin{tabular}{|l|c|c|}
\hline & Públicas & Privadas \\
\hline$\%$ de estudiantes que tienen ordenador propio & 65,4 & 78,2 \\
\hline $\begin{array}{l}\text { \% de universidades que exigen a sus estudiantes tener ordenador } \\
\text { propio }\end{array}$ & 3,2 & 14,3 \\
\hline
\end{tabular}

Tabla 4. Acceso al sistema de información en red para estudiantes en universidades norteamericanas. Fuente: Campus computing survey 2000. 
la propia institución como de preguntas formuladas por potenciales futuros estudiantes. Ello ha facilitado la creación de un considerable corpus de datos y estudios acumulados a lo largo de los últimos años. Esta situación contrasta con la escasez de la información disponible sobre el tema referente a las universidades de nuestro país. Algunas de nuestras universidades han creado ya sistemas de información en red para estudiantes tipo intranet muy completos e integrados, mientras que otras han empezado por poner en línea de forma dispersa algunos recursos de información.

\section{Notas}

1. Spillers-L. "What's in a cwis?". En: Campus wide information systems, 1994, v. 11, n. 3, pp. 19-32.

2. Campus wide information systems.

3. MIT Libraries. Annual report FY 1999-2000. Consultado en: 16-12-00. http://libraries.mit.edu/admin/ar00.html

4. Davenport, Thomas H. "Why we build systems that users won't use?". En: Computerworld, 1997, septiembre, v. 3, n. 2, pp. 2.

5. Twigg, Carol A.; Oblinger, Diana A. The virtual university. Consultado en: 25-11-00.

http://www.educause.edu/nlii/VU.html

6. Unesco. "Un programa europeu per al canvi en l'educació superior en el segle XXI: resultats del fòrum regional europeu, com a contribució a la conferència mundial sobre l'educació superior de la Unesco (Palermo, Italia, 24-27 de setembre de 1998)". En: Unesco. L'educació superior en el segle XXI. Palermo: Unesco, 1998, pp. 151-158.

7. Massy, W. F. "Life on the wired campus: how information technology will shape institutional futures". En: The learning revolution, Washington: Anker Publication, 1997, pp. 19-34.

8. Hutchinson, C. "The virtual university and the culture of learning". En: Educational libraries journal, 1998, v. 41, n. 1, pp. 5-11.

9. Forman, D. C. "The use of multimedia technology for training in business and industry". En: Multimedia monitor, 1995, v. 13, n. 7, 2.227 pp.

10. Verville, Anne-Lee. "What business needs from higher education". En: Educational record, 1995, v. 76, n. 4, pp. 46-50.

11. Pollock, N.; Cornford, J. "Theory and practice of the virtual university". En: Ariadne, 2000, enero.

12. Vigilante, R. The virtual college. New York: New York University, 1994.

13. Pittinsky, Matthew. "Studying today to envision tomorrow: the future of enterprise academic computing systems". En: Educause review articles, 1999.

14. Fathom. Consultado en: 30-11-00.

http://www.fathom.com

15. Sangrà, A.; Duart, J. M. Aprenentatge i virtualitat. Barcelona: Edicions de la Universitat Oberta de Catalunya, Pòrtic, 1999.

16. "100 most wired 2001". En: Yahoo internet life, 2001, octubre, pp. $101-102$.

17. Green, K. Campus computing 2000. Encino, CA, Campus Computing, 2001.

18. Educause. Educause consumer guide: how others are participating. Consultado en: 15-08-01.

http://www.educause.edu/consumerguide/others.html

19. Rosenfeld, L.; Morville, P. Information architecture for the world wide web. Sebastopol, CA: O'Reilly, 1998.

20. Gates, K. F. "Should colleges and universities require students to own their own computers?". En: Cause/Effect, 1998.
21. McRae, M. Online student services in American higher education: contemporary issues and future implications. North Texas University, 1999.

22. Nielsen, J. Useit. Consultado en: 15-07-01.

http://www.useit.com

23. Bernbom, G. "Institution-wide information management and its assessment". En: Information technology in higher education: assessing its impact and planning for the future. San Francisco: Jossey-Bass, 1999, pp. 71-84.

24. Heiralliance. Evaluation guidelines for institutional information resources. Consultado en: 13-08-00.

http://cause-www.colorado.edu/collab/heira.html

25. Fink, A. (ed.). The survey kit (v. 1-9). Thousand Oaks, CA: Sage, 1995.

26. McClure, Charles R.; Bertot, John Carlo. Evaluating networked information services: techniques, policy and issues. Maryland: Asis\&t, 2001.

27. Consejo de Universidades: Secretaría General. Catálogo de indicadores. Consultado en: 27-12-00.

http://www.mec.es/consejou/indicadores/anuncio.html

28. Green, K. Campus computing 2000. Encino, CA, Campus Computing, 2001.

29. "100 most wired 2001". En: Yahoo internet life, 2001, octubre, pp. 101-102.

30. McRae, M. Online student services in American higher education: contemporary issues and future implications. North Texas University, 1999.

31. Rebiun. Encuesta Rebiun 2000. Consultado en: 20-06-01.

http://www.uma.es/rebiun/InformeEstadistico.html

32. Lippincott, J. K. "Assessing the academic networked environment" En: Information technology in higher education: assessing its impact and planning for the future. San Francisco, CA: Jossey-Bass Inc., 1999, 21 pp.

33. Educause. Educause Information Resources Library. Consultado en: 20-03-01.

http://www.educause.edu/ir/ir-library.html

34. Universitat d'Alacant. Campus Virtual. Consultado 15-09-01. http//www.ua.es/va/univirtual

35. Universidad de Almería. Campus Virtual. Consultado 15-09-01. http://campus.ual.es/CAMPUS_VIRTUAL.HTM

36. Universitat Autònoma de Barcelona. Autònoma Interactiva. Consultado 15-09-01. http://www.uab.es/interactiva/default.htm

\section{Bibliografía}

Bernbom, Gerald (ed.). Information alchemy: the art and science of knowledge management. San Francisco, CA: Jossey-Bass Inc., 2001.

Educause. Educause consumer guide. Consultado en: 15-08-01. http://www.educause.edu/consumerguide/index.html

Katz, Richard N.; Rudy, Julia A. (eds.). Information technology in higher education. San Francisco, CA: Jossey-Bass Inc., 2000.

Luker, Mark A. (ed.). Preparing your campus for a networked future. San Francisco, CA: Jossey-Bass Inc., 2000.

McClure, Charles R.; Lopata, Cynthia L. Assessing the academic networked environment: strategies and options. Washington, D. C.: US Department of Education, 1996.

Josep Cobarsí Morales, Universitat Oberta de Catalunya. jcobarsi@uoc.edu 


\section{Nota de la Redacción}

EN EL PASADO NÚMERO de marzo-abril de 2002 de $E l$ profesional de la información (v. 11, n. 2, pp. 91-101) se publicó el artículo "Información parlamentaria autonómica en la Red: un impulso electrónico a la democracia", cuyas autoras son Mari Carmen Marcos y Ana Belén Gil.

La Redacción y el Consejo Asesor de EPI recibieron posteriormente un mensaje de Irene Ramos en el que se acusaba a las autoras de haber plagiado un artículo suyo, publicado conjuntamente con Miguel Ángel Gonzalo, "La documentación parlamentaria en Internet, II (el caso de las páginas web de las asambleas legislativas de las Comunidades Autónomas)". En: Revista de las Cortes Generales, 2000, n. 51, pp. 217-250.

Esta Dirección abrió inmediatamente una investigación para comparar los dos trabajos, escuchar las opiniones de ambas partes y consultar a varios expertos, tras lo cual ha obtenido el siguiente resultado:

Creemos que no estamos ante un caso de plagio (copiar una idea u obra de otro autor presentándola como si fuera propia), sino ante un evidente ejemplo de publicación redundante o duplicada; es decir, dos artículos que se solapan.

Las razones nos parecen claras:
1. Se trata de trabajos sobre el mismo tema y con los mismos objetivos: "...evaluación de las páginas web de los Parlamentos autonómicos" “...análisis de las páginas web de los parlamentos de las comunidades autónomas españolas...". Consideramos que las autoras del artículo publicado en EPI deberían haber realizado una búsqueda bibliográfica más exhaustiva previa a la iniciación de su trabajo para despejar la novedad del tema.

Una vez realizado el estudio, las autoras no ocultan su conocimiento del artículo pretendidamente plagiado sino que lo incluyen en su bibliografía, lo cual a nuestro entender avala su honestidad e integridad profesionales.

2. Se trata de trabajos que utilizan la misma metodología: el análisis de contenido de las páginas web a partir de una hoja de toma de datos basada en las directrices marcadas por la Unión Interparlamentaria (UIP) para páginas web parlamentarias:

http://www.ipu.org/english/pa rlweb.htm

http://www.ipu.org/cntre/web.pdf

cuyos criterios se dividen en tres bloques: ción;

-Contenidos o informa-

_Enlaces e interactividad;
—Facilidad de uso y diseño virtual.

Se utilizan los criterios de la UIP centrando la atención en tres aspectos:

a) información que se ofrece sobre la misma institución;

b) elementos que fomentan la participación ciudadana en la vida parlamentaria; y

c) herramientas que favorecen la accesibilidad a la información.

3. A pesar de que la fecha de publicación de la Revista de las Cortes Generales es del año 2000 , en realidad apareció mucho más tarde. Irene Ramos y Miguel Ángel Gonzalo realizaron el análisis en el mes de abril de 2001, y Mari Carmen Marcos y Ana Belén Gil lo realizaron a finales del año 2001, por lo que los periodos de toma de datos están separados tan sólo unos 6 meses.

\section{Conclusión}

Por todo ello, la Dirección de esta revista cree que se trata de trabajos realizados paralelamente (de hecho los resultados no son iguales), con una gran coincidencia derivada de la especificidad del objeto de estudio y de la utilización de unas recomendaciones internacionales comunes seguidas por ambos equipos, y que en ningún momento ha existido plagio.

Tomàs Baiget y Pedro Hípola

Directores de EPI 